\title{
Aerial Search Optimization Model (ASOM) for UAVs in Special Operations
}

\author{
Moshe Kress and Johannes O. Royset \\ Operations Research Department \\ Naval Postgraduate School
}

March 9, 2007

\begin{abstract}
We construct an optimization model that assists commanders, operators, and planners to effectively deploy and employ unmanned aerial vehicles (UAVs) in special operations missions. Specifically, we consider situations where targets (e.g., insurgents) operate in a region of interest and a small special operations team is assigned to search and detect these targets. The special operations team is equipped with short-range surveillance UAVs. We combine intelligence regarding the targets with availability and capability of UAVs in an integer linear programming model. The goal is to detect the largest possible number of targets with the given resources. The model prescribes optimal deployment locations for the ground units and optimal time-phased search areas for the UAVs. The model has been implemented successfully in four field experiments. Preliminary empirical evidence indicates that the model provides $50 \%$ increase in detection opportunities compared with a plan manually generated by experienced commanders.
\end{abstract}

\section{Introduction}

Special operations missions are expected to increasingly make use of unmanned aerial vehicles (UAVs) for reconnaissance, surveillance, search, and enhanced situational awareness (Livingroom 2006, Feickert 2006, and Rolfsen 2005). However, concepts of operations for the use of UAVs in this context have not been fully developed (Cross 2006). In this paper we address the problem of optimally utilizing UAVs by small teams of special operations forces (SOF) in a typical scenario. Specifically, we consider a situation where mobile targets (e.g., insurgents) operate in or transit through a certain region of interest. A SOF team, controlled by a tactical operations center, operates several short-range surveillance UAVs to search for the targets. Each UAV is controlled by a 
ground control unit (GCU) deployed also in the region. Surveillance data from a UAV is collected by its GCU and transmitted, through a mobile control center (MCC), to the tactical operations center. The MCCs are needed as relays to overcome short communication ranges of GCUs, and possible limited line-of-sight between a GCU and the tactical operations center. The problem is how to deploy the ground units (GCUs and MCCs) and how to route and schedule the UAVs in a most effective way.

In response to operational needs, and in close consultation with SOF commanders, UAV operators, and military air traffic controllers, we have developed an integer linear programming model that prescribes optimal deployment locations for the MCCs and GCUs, and assigns optimal search areas and schedules for the UAVs. The model combines (partially uncertain) information about the availability of UAVs with intelligence, of two types, about the targets. The model has been utilized as a planning tool in four multi-day field experiments carried out at Camp Roberts, California, under the USSOCOM-NPS Cooperative Field Experimentation Program. Empirical results from these experiments show a 50\% increase in detection opportunities compared with a plan manually generated by experienced commanders. The operational setting in these experiments and the type of scenarios played lead us to believe that this increase of performance may be a conservative estimate for the actual increase in search effectiveness in real-life operations.

To the authors' knowledge, the problem of determining deployment locations for MCCs and GCUs, and time-phased optimal search areas for UAVs has not been examined systematically in the open literature. There are however several related problems that have been studied in the past. In its simplest form, the problem of routing one UAV over points of interest can be formulated as an orienteering problem (also known as the maximum collection and selective traveling salesman problems) (Feillet et al. 2005). The orienteering problem is defined on a network where each node represents a point of interest and each arc represents travel between two nodes. Each node is associated with a prize and each arc with a travel time. The goal is to find a maximum-prize path or cycle whose total travel time does not exceed a specific limit. In the context of UAV search, the 
prize at a node may be an additive surrogate of the probability of detecting a target at the corresponding point of interest. In search and reconnaissance, a visit to certain points of interest may only be allowed during specific time windows and the prize associated with a visit may be time dependent. Generalizations of the orienteering problem to cases with time-window constraints have been considered; see Kantor and Rosenwein (1992) for a theoretical study. Models with time dependent prizes are found in Brideau and Cavalier (1994) and Erkut and Zhang (1996), which consider a competitive environment where sales may decline over time due to the operation of competing salespersons.

In many situations the mission is to route multiple UAVs simultaneously. Chao et al. (1996) consider an extension of the orienteering problem that models the sport of team orienteering, where the combined prize-collecting effort of a team of participants is maximized. Millar and Kiragu (1997) consider a similar multi-vehicle orienteering problem with application to dispatching of fishing patrols. A generalization of the orienteering problem to multiple vehicles with time-window constraints is considered in Moser (1990), which routes aerial reconnaissance assets in a military setting. This model assigns a time-invariant prize to the visit of each point of interest. Moser (1990) addresses a similar problem to ours, but does not account for important factors such as airspace deconfliction constraints, time-dependent intelligence and ground-units' deployment flexibility. We find similar shortcomings in Eagle and Yee (1990), Dell et al. (1996), and Washburn (1998), which consider a target that moves between cells according to some probability law, and one or more searchers that follow a continuous path searching for the target. These three papers derive specialized branch-and-bound algorithms for solving these problems optimally. Dell et al. (1996) also devise six heuristic algorithms.

Our problem of determining deployment locations for ground units and time-phased search areas for UAVs also relates to location-routing problems in manufacturing and distribution industries (Laporte 1988 and Min et al. 1998). In these problems, a strategic level decision regarding facility locations is made before the operational decisions regarding routing of vehicles between facilities and customers. This "two-stage" decision 
process of location-routing problems motivates our approach, but the specific details of UAV operations hinder direct application of existing location-routing models.

Our model extends previous work in this area in several dimensions. First, we consider the two decisions regarding ground units' locations and UAVs' time-phased search areas within the same model. These two decisions are strongly connected by factors such as UAV range, topography, and communication capabilities. Second, operational constraints such as deconfliction among UAVs, flight endurance and possible communication interferences are explicitly represented in the model. Third, the model takes into account two types of uncertain information: operational readiness of UAVs and intelligence regarding possible threat scenarios. The uncertainties are reduced as the scenario unfolds, and by defining two levels of that uncertainty, we derive a two-stage optimization model. None of the existing models in the literature address all three aspects.

In Section 2, we describe the combat situation in more detail. Section 3 presents our optimization model and Section 4 discusses a specific case study that illustrates the implementation of the model as a planning tool.

\section{The Combat Situation}

Consider a situation where intelligence sources indicate that targets are expected to enter the region of interest in the near future. A special operations team, consisting of MCCs, GCUs, and UAVs of various types, is deployed in the region with the mission to search and detect the targets. Intelligence reports and analyses of past events indicate possible types of targets (e.g., on foot, in vehicles, etc.) and potential entry areas into the region. These reports also give good estimates for the velocities of the various types of targets. Each entry area and target type is associated with a number of routes that a target may take from the entry area. This information is called henceforth general intelligence. The general intelligence includes also the probabilities of a type of target and entry area, and the conditional probabilities of the routes associated with a certain entry area. 
Small UAVs are not as reliable and weather-robust as manned aircraft and therefore may not always be mission ready when called upon. It follows that the number and mix of mission-ready UAVs available at the start of a search mission are not known with certainty until the UAVs are about ready to be launched. Thus, we assume that the number of mission-ready UAVs of a certain type is a random variable with a known probability mass function estimated from past readiness data. The general intelligence and the readiness data generate scenarios; each scenario comprises an entry area, a set of targets and a mix of mission-ready UAVs. We assume a finite set of scenarios, each with a known probability of occurrence, which is obtained from the general intelligence and past readiness data.

The UAVs are launched once the team gets a clear indication (based on human intelligence or interception of communication) that one or more targets have entered the region. At that time, the entry areas and the number and type of targets become known, but the route selected remains unknown. We refer to the newly arrived information regarding the time and area of entry, and the number and type of targets, as specific intelligence. When the specific intelligence arrives, the search mission starts. At this time, the availability of mission-ready UAVs becomes known too. In other words, the realized scenario becomes known. Note that while the mission is planned and the ground units are deployed according to the probability distribution of the scenarios, which is based on the general intelligence and the uncertain readiness of the UAVs, the mission starts and the UAVs are launched only when the realized scenario unfolds, following the arrival of the specific intelligence.

Each UAV has its own GCU, with which it must maintain constant line-of-sight and be within a certain range to avoid loss of control and interruption in data transfer. A UAV is launched from a location near its GCU and, while airborne, searches designated areas in an attempt to detect targets. The UAVs are equipped with electro-optical and infrared sensors. To avoid airspace conflicts and possible accidents, every UAV must maintain a minimum distance to other UAVs at all times. This deconfliction is achieved by assigning different altitudes or different non-overlapping search areas to the UAVs. Every GCU 
must be located sufficiently close to an MCC so it can be connected to the MCC by either a cable or a wireless transmission. Moreover, due to interference between GCUs, a minimum distance, dependent on the type of GCU, must be maintained between any two GCUs. The GCUs and MCCs are mobile, but must be stationary when their controlled UAVs are airborne. The MCCs and the GCUs require a substantial setup time to redeploy, which comprises collecting the GCUs and UAVs, packing and moving the equipment, re-deploying the ground units, and checking communication among the tactical operations center, the MCCs, and the GCUs. In this study we consider a relatively short planning horizon of 1-10 hours, which, in practice, prevents effective redeployment. Hence, we assume that the MCCs, and their associated GCUs, remain stationary throughout the planning horizon.

There are several types of UAVs available for the mission, which differ in their cruising altitude, field-of-view, resolution of their sensor, and velocity. We consider only spatial deconfliction requirements, which apply only to UAVs that share the same cruising altitude. Communication frequency conflicts may also occur between two wireless transmissions. While accounting for this effect in the implementation of our model, for simplicity of exposition it is ignored in this paper.

The goal of our model is to assist commanders in determining suitable deployment locations for MCCs and GCUs, and optimal time-phased search areas for the UAVs. In the next section, we describe an optimization model that achieves this goal and that takes into account operational constraints such as air space deconfliction, line-of-sight, communication ranges, air velocity, and flying endurance.

\section{Model Formulation}

We discretize the planning horizon into time steps $t \in T=\{1,2, \ldots,|T|\}$, each of a fixed duration $\Delta$. Let $U$ be the set of UAVs available to the special operations team, where each UAV $u \in U$ has flying endurance $e_{u}$ measured in time steps. For each $u \in U$, we divide the region of interest into a set $A_{u}$ of non-overlapping search areas, where 
$A_{u}=A_{u^{\prime}}$ if UAVs $u$ and $u^{\prime}$ are of the same type. A set $A_{u}$ may cover the whole region of interest or only sub-regions of particular interest such as road segments, trails, and villages. The length of the time steps and the size of the search areas are selected based on the UAVs' speed, altitude, sensor capabilities, and other factors, as discussed in Section 4 . We also specify a finite set $L$ of potential locations for deploying the MCCs and a finite set $G$ of possible locations for deploying the GCUs. At any time step $t$, a UAV $u \in U$ is on the ground at its GCU site or is flying over an area $a \in A_{u}$ searching for targets.

The planning process of the UAVs' mission is divided into two stages. First, the objective is to determine the locations of the MCCs and the GCUs based on the general intelligence and the uncertain readiness of the UAVs, that is, based on the possible scenarios and their probabilities. This decision is referred to as the first-stage decision. As described in Section 2, the locations of the MCCs and GCUs must be determined several hours prior to commencement of the UAV search operation. After the MCCs and GCUs are deployed and are ready to launch their UAVs, and the specific intelligence about enemy activity and entry areas becomes available, the second-stage decision regarding the UAVs' time-phased search areas is made and executed. Specifically, the second-stage decision, which is made after the realized scenario becomes known, determines the search area for each UAV at every time step $t \in T$ of the operation. Note that while the uncertainty regarding the number and types of targets, the time and area of entry, and the readiness of UAVs is resolved when making the second-stage decision, the information regarding the specific routes taken by the targets in the realized scenario remains uncertain.

While our model can handle multiple targets, for simplicity of exposition we consider only one target entering the region of interest. Let $S$ be a finite set of scenarios. Recall that a scenario $s \in S$ is defined by the type of target, the area of entry, and the mix of mission-ready UAVs. Each scenario $s \in S$ occurs with a probability $q_{s}$. Given a scenario $S$, there is a set of routes, denoted $R_{s}$, that the target may take to traverse the region. Let 
$p_{s, r}, r \in R_{s}$, denote the (conditional) probability that the target follows route $r$, given scenario $s$. These probabilities are estimated based on tactical intelligence information regarding the likelihood of the various routes. In principle, this information may be updated during the course of the operation based on new intelligence that arrives and the results of the UAV search. Such possible intelligence updates lead to multi-stage models, which are beyond the scope of this paper.

Given a scenario s, which includes the type of target and hence its velocity, and a route $r \in R_{s}$, we define the fractional presence by:

$$
\begin{aligned}
\tau_{a, s, r}(t)= & \text { fraction of time step } t \in T \text { the target spends in } \\
& \text { search area } a \text { given scenario } s \in S \text { and route } r \in R_{s} .
\end{aligned}
$$

For example, suppose that a time step is $\Delta=10 \mathrm{~min}$. According to scenario $s$ and route $r \in R_{s}$, the target is going to be in search area $a$, between time (measured in minutes) 17 and 25 from the beginning of the planning horizon. Then, $\tau_{a, s, r}(1)=0$, $\tau_{a, s, r}(2)=0.3, \tau_{a, s, r}(3)=0.5$, and $\tau_{a, s, r}(t)=0$ for $t=4,5, \ldots$. Based on the values of the fractional presence $\tau_{a, s, r}(t)$, we can compute the "reward" of employing UAVs in certain search areas. There are several possible approaches for computing such a reward function, as discussed in the following.

Let $v_{u}$ be the ground speed and $w_{u}$ the sweep width of a cookie-cutter sensor mounted on UAV $u$. Also, let $d_{a}$ be the area size of search area $a$. Then, assuming random search within a search area, the conditional probability, given scenario $s$ and route $r$, for the target evading all attempts by UAV $u$ searching area $a$ during time period $t$ is $\exp \left(-\Delta \tau_{a, s, r}(t) w_{u} v_{u} / d_{a}\right)$ (see, e.g., Washburn 2002). Let $X_{u, a, t, s}$ be a binary variable, with $X_{u, a, t, s}=1$ if UAV $u$ is searching area $a$ during time step $t$ in scenario $s$, and $X_{u, a, t, s}=0$ otherwise. Note that $X_{u, a, t, s}$ are second-stage decision variables (also called recourse variables). Assuming independent detections among UAVs and among the various time steps, the conditional probability, given scenario $s$ and route $r$, of the target evading all UAVs during the whole planning horizon is 


$$
\prod_{u \in U} \prod_{a \in A_{u}} \prod_{t \in T} \exp \left(-\Delta \tau_{a, s, r}(t) w_{u} v_{u} X_{u, a, t, s} / d_{a}\right) .
$$

In view of (1), the (unconditional) probability of the target evading all UAVs during the planning horizon is

$$
\sum_{s \in S} \sum_{r \in R_{s}} q_{s} p_{s, r} \prod_{u \in U} \prod_{a \in A_{u}} \prod_{t \in T} \exp \left(-\Delta \tau_{a, s, r}(t) w_{u} v_{u} X_{u, a, t, s} / d_{a}\right) .
$$

Ideally, we would like to adopt this probability measure as the objective function and to minimize it. However, due to its nonlinearity, and in anticipation of a large number of linear constraints, we opt to use a linear surrogate for (2) so that the problem formulation remains linear. In the following we present four possible linear objective functions as surrogates for (2).

\section{Worst-case Scenario and Route}

Instead of minimizing (2), we minimize the largest probability of evasion over all scenarios and routes, i.e., we minimize

$$
\max _{s \in S, r \in R_{s}} \prod_{u \in U} \prod_{a \in A_{u}} \prod_{t \in T} \exp \left(-\Delta \tau_{a, s, r}(t) w_{u} v_{u} X_{u, a, t, s} / d_{a}\right) .
$$

Using a standard logarithmic transformation, this objective function is linearized by maximizing an auxiliary variable $\xi$ subject to the constraints

$$
\sum_{u \in U} \sum_{a \in A_{u}} \sum_{t \in T} \Delta \tau_{a, s, r}(t) w_{u} v_{u} X_{u, a, t, s} / d_{a} \geq \xi \quad \forall s \in S, r \in R_{s} .
$$

Note that the worst-case formulation does not depend on the scenario and route probabilities. 
Worst-case Scenario and Expected Fractional Presence

This surrogate is similar to (3) but considers only scenarios, not individual routes. It minimizes the largest probability of evasion over all scenarios and utilizes the expected scenario fractional presence defined by

$$
\bar{\tau}_{a, s}(t)=\sum_{r \in R_{s}} p_{s, r} \tau_{a, s, r}(t)
$$

This leads to the following objective function

$$
\max _{s \in S} \prod_{u \in U} \prod_{a \in A_{u}} \prod_{t \in T} \exp \left(-\Delta \bar{\tau}_{a, s}(t) w_{u} v_{u} X_{u, a, t, s} / d_{a}\right) .
$$

Again, using a standard logarithmic transformation, the problem is to maximize an auxiliary variable $\xi$ subject to the constraints

$$
\sum_{u \in U} \sum_{a \in A_{u}} \sum_{t \in T} \Delta \bar{\tau}_{a, s}(t) w_{u} v_{u} X_{u, a, t, s} / d_{a} \geq \xi \quad \forall s \in S
$$

\section{Average Overlap Time}

This surrogate maximizes the average overlap time between UAVs and the target. It represents the "detection opportunities" of the UAVs. In this case, we maximize

$$
\sum_{t \in T} \sum_{u \in U} \sum_{a \in A_{u}} \sum_{s \in S} q_{s} \Delta \bar{\tau}_{u, a, s}(t) X_{u, a, t, s} .
$$

Note that (8) does not account for the capabilities (sweep-width and velocity) of the various UAV and only attempts to place the UAVs in the "right" area at the "right" time.

\section{Linearized Evasion Probability}


We also derive a lower bound on the probability of evasion (2), which we use as a surrogate of that probability. Using the fact that $\exp (-z) \geq 1-z$ for real-valued $z$, we obtain that

$$
1-\sum_{s \in S} \sum_{r \in R_{s}} \sum_{u \in U} \sum_{a \in A_{u}} \sum_{t \in T} q_{s} p_{s, r} \Delta \tau_{a, s, r}(t) w_{u} v_{u} X_{u, a, t, s} / d_{a} .
$$

is a lower bound on (2). We note that minimizing (9) is identical to maximizing (8) with the exception of the constant $w_{u} v_{u} / d_{a}$ in (9). In the case study of Section 4, we use (9) as the objective function.

In the following formulation of the complete model, we let $f(X)$ be a generic objective function in the form (2), (3), (6), the negative of (8), or (9) that we seek to minimize. Multiple optima may occur in these optimization problems, some of which may result in frequent and unnecessary changes of search areas. To eliminate such solutions, we assign a small penalty $\varepsilon$ to each change of search area and add the total penalty to the objective function.

Based on these assumptions, we formulate a two-stage integer linear stochastic program with recourse, where the first-stage decision variables determine the locations of the MCCs and GCUs, and the second-stage decision variables specify the time-phased search areas for the UAVs given the realized scenario. We note that there is no reward associated with the first-stage decision.

\section{Notation}

Indices

$\begin{array}{ll}u, u^{\prime} & \text { UAV or its GCU, } u \in U . \\ t & \text { Time step, } t \in T . \\ a, a^{\prime} & \text { Search area, } a \in \bigcup_{u \in U} A_{u} \cdot \\ s & \text { Scenario, } s \in S . \\ m & \text { Mobile control center (MCC), } m \in M .\end{array}$


$g, g^{\prime} \quad$ Ground control unit (GCU) locations, $g \in G$.

Sets

$C(u, a) \quad$ UAV and search area pairs $\left(u^{\prime}, a^{\prime}\right)$, with $u^{\prime} \in U$ and $a^{\prime} \in A_{u^{\prime}}$, such that UAV $u^{\prime}$ cannot search area $a^{\prime}$ at the same time step UAV $u$ is searching area $a$.

$N(u, a) \quad$ Subset of search areas $A_{u}$ that UAV $u$ can move to from search area $a$.

$L(u, g) \quad$ Subset of MCC locations $L$ that allows connection between the MCC and GCU $u$ at location $g$.

$G(u, a) \quad$ Subset of GCU locations $G$ from which UAV $u$ can search area $a$.

$H(u, g) \quad$ GCU and location pairs $\left(u{ }^{\prime}, g^{\prime}\right)$ such that GCU $u^{\prime}$ cannot be located at $g^{\prime}$ if GCU $u$ is located at $g$.

\section{Data}

$\varepsilon$

$e_{u}$

$q_{s}$

$p_{s, r}$

Variables

$X_{u, a, t, s}$

$Y_{m, l}$

$Z_{u, g}$

$W_{u, t, s}$
Transition penalty for each UAV.

Endurance of UAV u (time steps).

Probability of scenario $s$.

Probability of route $r$ given scenario $s$.

Mathematical Formulation 


$$
\min f(X)+\sum_{s \in S} q_{s} \sum_{u \in U} \sum_{t \in T} \varepsilon W_{u, t, s}
$$

s.t.

$$
\begin{aligned}
& \sum_{a \in A_{u}} X_{u, a, t, s} \leq 1, \quad \forall u, t, s \\
& \sum_{l \in L} Y_{m, l}=1, \quad \forall m \\
& \sum_{\left(u^{\prime}, a^{\prime}\right) \in C(u, a)} X_{u^{\prime}, a^{\prime}, t, s} \leq \min \{|U|-1,|C(u, a)|\}\left(1-X_{u, a, t, s}\right), \quad \forall u, a \in A_{u}, t, s \\
& X_{u, a, t, s} \leq X_{u, a, t+1, s}+\sum_{a^{\prime} \in N(u, a)} X_{u, a^{\prime}, t+1, s}, \quad \forall u, a \in A_{u}, t \leq|T|-1, s \\
& Z_{u, g} \leq \sum_{m \in M} \sum_{l \in L(u, g)} Y_{m, l}, \quad \forall u, g \\
& \sum_{g} Z_{u, g}=1, \quad \forall u \\
& \sum_{u} Z_{u, g} \leq 1, \quad \forall g \\
& \sum_{\left(u^{\prime}, g^{\prime}\right) \in H(u, g)} Z_{u^{\prime}, g^{\prime}} \leq \min \{|U|-1,|H(u, g)|\}\left(1-Z_{u, g}\right), \quad \forall u, g \\
& X_{u, a, t, s} \leq \sum_{g \in G(u, a)} Z_{u, g}, \quad \forall u, a \in A_{u}, t, s \\
& \sum_{t^{\prime}=t}^{t+e_{u}} \sum_{a \in A_{u}} X_{u, a, t^{\prime}, s} \leq e_{u}, \quad \forall u, t \leq|T|-e_{u}, s \\
& X_{u, a, t, s}-X_{u, a, t-1, s} \leq W_{u, t, s}, \quad \forall u, a \in A_{u}, t \geq 2, s \\
& X_{u, a, t, s}, Y_{m, l}, Z_{u, g}, W_{u, t, s} \in\{0,1\}, \quad \forall u, a, t, s, l, m, g
\end{aligned}
$$


Constraints (10) ensure that each UAV searches at most one search area during a time step. In constraints (11), we select one location for each MCC. Air-space deconfliction is manifested in constraints (12), where a UAV is prevented from searching areas that are in conflict with other UAVs' search areas. Constraints (13) ensure feasible flying routes; each UAV either remains in the same search area or moves to an adjacent area in the next time step. We assume that there is no transit time between adjacent search areas, otherwise we could simply define dummy search areas. Constraints (14) determine the feasible deployment locations for the various GCUs. Constraints (15) assure that each GCU is assigned to one location only, and constraints (16) specify that from a certain deployment location at most one GCU can operate. Constraints (17) represent GCU deconfliction requirement and constraints (18) restrict UAVs' searches only to areas controllable from their respective GCU locations. Constraints (19) limit the number of time steps in which UAVs can be airborne. Constraints (20) ensure that $W_{u, t, s}=1$ if UAV $u$ transitions to a new search area in time step $t$. Each transition is penalized in the objective function to avoid unnecessary transitions.

\section{Case Study}

During 2006, several versions of this model were implemented in four, multi-day field experiments at Camp Roberts, California. These field experiments involved up to 16 UAVs searching for one or more targets in sets of controlled, yet uncertain, situations. The goal of these field experiments was to implement and test, in cooperation with UAV operators and commanders, the optimization model in a real-world, operational setting. The model provided optimal deployment sites for ground units and time-phased search areas for UAVs that were executed in the field experiments. In the following, we describe in detail the implementation of the model for the October 29-31, 2006 field experiment. In this field experiment we compared the effectiveness of an optimal search plan generated by our model with a plan formulated by experienced commanders and operators.

Operational Setting and Implementation 
A Special Operations team is assigned three UAVs: a Tern (Tern 2007), a BUSTER (Buster 2007), and a Raven (Raven 2007), each with its corresponding GCUs, and one MCC. Even though all three UAVs are physically present at the staging area, past experience indicates that the Tern, BUSTER, and Raven can be successfully launched only $80 \%, 70 \%$, and $60 \%$ of the time, respectively. According to general intelligence reports, a target may enter the region of interest within a few hours. Consequently, the team deploys its ground units - GCUs and MCC - to certain locations so that it can rapidly and effectively respond to alerts generated by a specific intelligence report. This report indicates an imminent entry of a specific target into the region of interest and a corresponding entry area. Upon receiving the specific information regarding the target, the UAVs are launched to search and detect the target. The region of interest is Camp Roberts, a California National Guard base in California's Central Coast region. According to general intelligence, the target may enter the region of interest through one of six possible entry areas labeled E1, E2, S1, S2, W1 and W2, see Figure 1. The target is assumed to be a vehicle. The six entry areas, coupled with seven possible non-empty mixes of the three UAVs, generate 42 scenarios. Once the specific intelligence becomes available, one of these 42 scenarios is realized and the search operation begins. The remaining uncertainty is with regard to the route the target selects from the given entry area.

The planning horizon is 48 minutes, starting at the target's time of entry, and consists of six 8-minute time steps (i.e., $\Delta=8 \mathrm{~min}$ ). The length of a time step has been determined after consulting UAV operators. This length is a compromise between increasing the resolution and sensitivity of the model and the desire to avoid overloading the commandand-control system with too frequent changes in search areas. We assume that processing the specific intelligence and preparing the UAVs for take-off consume one time step, so the UAVs are available for search during time steps 2-6.

The probabilities of the 42 scenarios are evaluated as follows. Each one of four entry areas (in the west and east of Camp Roberts) has probability 1/8 of occurring, while each of the two entry areas in the south has probability $1 / 4$. These probabilities, together with 
the probabilities of mission-ready mixes of UAVs, determine the scenario probabilities $q_{\mathrm{s}}, s \in S$. The region of interest contains numerous roads. From an entry area, the target moves on these roads, along a minimum-time route, either towards an exit zone in the northeast or towards one of 11 possible internal destination points, labeled C1,..,C11 in Figure 1. The target moves at 10 and 15 miles per hour on dirt and paved roads, respectively. General intelligence estimates that the target will proceed to the exit zone with probability 0.5 . Otherwise it will move towards one of the internal destination points. If the target exits the area, it cannot be detected anymore. If the target reaches an internal destination point, it remains stationary and subject to detection. All of the internal destination points are assumed to be equally likely. From this information, we generate a set of twelve possible routes $R_{s}$ for the target in each scenario, with corresponding probabilities of occurrence $p_{s, r}$.

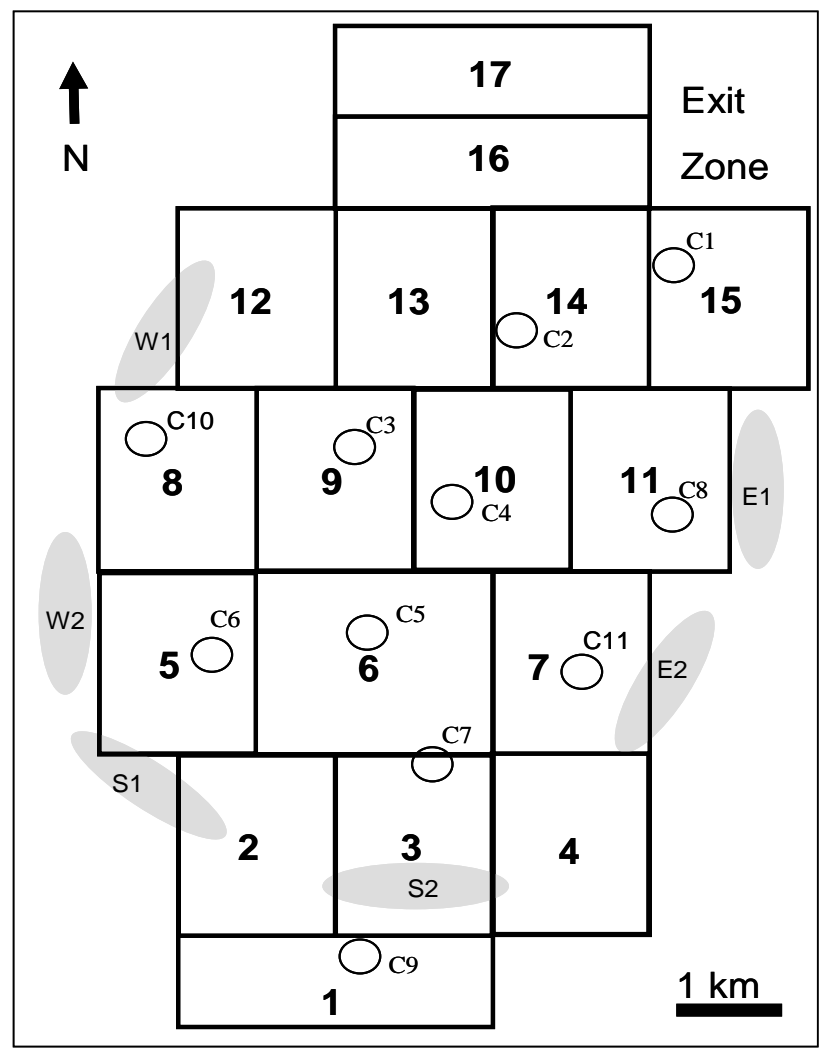

Figure 1: Entry Areas (ovals), Destination Points (circles), and Area Cells (boxes) in the region of interest at Camp Roberts 
The region of interest is divided into 17 area cells, see Figure 1. Each area cell represents one search area for the Raven. Two or three area cells are grouped together to form appropriate search areas for BUSTER and Tern. These UAV-dependent sets of area cells, denoted $A_{u}$, are designed according to the speed, maneuverability and sweep width of the specific UAV.

All UAVs have endurance longer than 40 minutes and hence the constraints in (19) are redundant. With this data, the model is implemented in GAMS and is solved using CPLEX 10.0. The model consists of approximately 30,000 variables and 60,000 constraints. The total solution time is 2 minutes on a $3.8 \mathrm{GHz}$ desktop computer with 3 GB of RAM. The output of the model is optimal locations for the MCC and GCUs and, for each scenario, optimal time-phased search areas for the UAVs.

\section{Results}

Table 1 presents an example of the model output for entry area W1 in the case of all UAVs being available. Each row in Table 1 specifies the designated area cells for the UAVs as defined in Figure 1. We note that Raven is always assigned a single area cell, while BUSTER and Tern searches multiple area cells in the same time period due to their higher speed and altitude. To compare, we also asked a group of experienced UAV operators and commanders to plan search areas for the given scenarios based on the general intelligence, the UAV readiness data, and the optimal location of the ground units, provided to them from the model solution. Their resulting plan assigned each UAV to a certain sub-area of the region of interest throughout the operation.

We randomly generated 36 situations from the 504 possible situations (42 scenarios times 12 target routes from each entry area). These 36 situations were implemented during the experiment at Camp Roberts both according to the model's optimal plan and the manual 
plan generated by the commanders. The order of the exercises was randomized to avoid biases due to operator errors, day light conditions, etc.

Our model does not account for possible loss of video link, poor visibility, and other factors that may prevent a sensor to detect a target, given that the target is in the sensor's field of view. Furthermore, the model does not deal with target recognition and identification. Consequently, we counted the number of detection opportunities situations where the target is in the UAV's vicinity - as the measure of effectiveness (MOE). Since both the target and the UAVs were continuously tracked, this MOE could be calculated quite reliably. In 24 of the 36 situations exercised using the model as the planning tool, a detection opportunity of the target was recorded. The corresponding number when using the manual plan was 16 detection opportunities. Hence, our model increased the probability of having a detection opportunity by $50 \%$ - from $44 \%$ in the manual plan to $67 \%$.

\begin{tabular}{|c|c|c|c|}
\hline \multirow{2}{*}{ Time Period } & \multicolumn{3}{|c|}{ Area Cells } \\
\cline { 2 - 4 } & Raven & BUSTER & Tern \\
\hline $0-8$ min & At GCU & At GCU & At GCU \\
\hline $8-16$ min & 6 & $16 ; 17$ & $12 ; 13$ \\
\hline $16-24$ min & 10 & $16 ; 17$ & $8 ; 9$ \\
\hline $24-32$ min & 11 & $12 ; 13$ & $5 ; 6$ \\
\hline $32-40$ min & 7 & $14 ; 15$ & $10 ; 11$ \\
\hline $40-48$ min & 7 & $10 ; 11$ & $14 ; 15$ \\
\hline
\end{tabular}

Table 1. Optimal search plan (given in terms of area cells, see Figure 1) in the case of W1-entry by the target.

\section{Conclusions}

We have developed a two-stage stochastic integer linear programming model for optimizing UAV deployment and employment during special operations search missions. 
The model determines optimal locations of ground control units and mobile control centers, as well as time-phased search areas for UAVs. We ensure that the output of the model is robust with respect to a variety of contingencies by accounting for (uncertain) information about target movement as well as reliability of the available UAVs. The model has been utilized by commanders, UAV operators, and military air traffic controllers as a planning tool during four field experiments at Camp Roberts, California. Comparing the optimized plan with manual plans generated by experienced commanders, the model provided plans that resulted in 50\% more detection opportunities of targets. We note that commanders are not used to plan search missions with a mix of different UAVs, which explains part of this improvement. However, even for trained commanders, the large number of constraints related to air space deconfliction, line-of-sight restrictions and other physical and operational conditions, may be overwhelming. These constraints, coupled with ambiguous intelligence pictures, make manual planning tedious, error prone, and most likely - sub-optimal. A model like ASOM, which has been described in this paper, can prove to be a valuable and useful decision support system for UAV search missions.

\section{Acknowledgements}

The authors are grateful to Dr. David Netzer, Naval Postgraduate School, and USSOCOM for suggesting this research project and supporting it. The authors also greatly appreciate the invaluable input received from Dr. David Netzer, Commander Gordon Cross, U.S. Navy, Lieutenant Colonel Kenneth Paxton, U.S. Air Force, Mr. Samuel Nickels, U.S. Air Force, and Lieutenant Colonel Mark Brinkman, U.S. Marine Corps. The authors thank Major Daniel Reber, U.S. Marine Corps, and Mr. Michael Clement for their assistance in preparing and recording the scenarios.

\section{References}

R.J. Brideau III and T.M. Cavalier, 1994. The maximum collection problem with time dependent rewards. In Proceedings of TIMS International Conference, Anchorage, Alaska. 
Buster, 2007. Designation Systems website. Accessed January 10.

www.designation-systems.net/dusrm/app4/buster.html

I.M. Chao, B.L. Golden, and E.A. Wasil, 1996. The team orienteering problem. European Journal of Operational Research, 88(3):464-474.

G. Cross, 2006, Commander, U.S. Navy, Private communication, October.

R. F. Dell, J. N. Eagle, G. H. A. Martins, and A. G. Santos, 1996. Using Multiple Searchers in Constrained-Path Moving-Target Search Problems. Naval Research Logistics, 43: 463-480.

J. N. Eagle and J. R. Yee, 1990. An Optimal Branch-and-Bound Procedure for the Constrained Path Moving Target Search Problems. Operations Research, 38(1): 110-114.

E. Erkut and J. Zhang, 1996. The maximum collection problem with time-dependent rewards. Naval Research Logistics, 43(5):749-763.

A. Feickert, 2006. US Special Operation Forces (SOF): Background and Issues for Congress, CRS Report for Congress, Order Code RS21048, April.

D. Feillet, P. Dejax, and M. Gendreau, 2005. Traveling salesman problems with profits. Transportation Science, 39(2):188-205.

M.G. Kantor and M.B. Rosenwein, 1992. The orienteering problem with time windows. Journal of the Operational Research Society, 43(6):629-635.

G. Laporte, 1988. Location-routing problems. In B.L. Golden and A.A. Assad (Eds.), Vehicle routing: methods and studies, North Holland, Amsterdam.

Livingroom website, 2006. Accessed August 1.

www.livingroom.org.au/uavblog/archives/special_operations_command_get_predator_uav_squadron.php 
H. H. Millar and M. Kiragu, 1997. A time-based formulation and upper bounding scheme for the selective traveling salesperson problem. Journal of the Operational Research Society, 48(5): 511-518.

H. Min, V. Jayaraman, and R. Srivastava, 1998. Combined location-routing problems: a synthesis and future research directions. European Journal of Operational Research, 108:1-15.

H.D. Moser, 1990. Scheduling and routing tactical aerial reconnaissance vehicles. Master's thesis, Naval Postgraduate School, Monterey, California.

Raven, 2007. Global Security website. Accessed January 10. http://www.globalsecurity.org/intell/systems/raven.htm

B. Rolfsen, 2005. Special Operations Predators, Armed Forces Journal, July, pp. 18-19.

Tern, 2007. Designation Systems website. Accessed January 10.

www.designation-systems.net/dusrm/app4/tern.html

A. R. Washburn, 1998. Branch and Bound Method for a Search Problem. Naval Research Logistics, 45:243-257.

A. R. Washburn, 2002. Search and Detection, 4th Ed., INFORMS, Linthicum, Maryland. 